\title{
Polysèmes
}

Revue d'études intertextuelles et intermédiales

\section{Le détour par le pictural dans The Virgin in the Garden et Still Life de A.S. Byatt}

Émilie Bourdarot

\section{(2) OpenEdition}

Journals

Édition électronique

URL : http://journals.openedition.org/polysemes/623

DOI : $10.4000 /$ polysemes.623

ISSN : 2496-4212

Éditeur

SAIT

Édition imprimée

Date de publication : 1 janvier 2008

Pagination : 29-42

ISSN : 0999-4203

\section{Référence électronique}

Émilie Bourdarot, "Le détour par le pictural dans The Virgin in the Garden et Still Life de A.S. Byatt », Polysèmes [En ligne], 10 | 2008, mis en ligne le 01 mars 2015, consulté le 19 avril 2019. URL : http:// journals.openedition.org/polysemes/623 ; DOI : 10.4000/polysemes.623

Ce document a été généré automatiquement le 19 avril 2019

Polysèmes 


\title{
Le détour par le pictural dans The Virgin in the Garden et Still Life de A.S. Byatt
}

\author{
Émilie Bourdarot
}

1 Le « détour » par le pictural dans les œuvres de Byatt est le corollaire d'une écriture qui mêle réalisme et réflexions métafictionnelles, forme hybride que l'écrivain nomme « selfconscious realism", et qui ne cesse d'interroger le statut et les formes de la représentation. Son écriture très sensuelle et les multiples considérations théoriques qui ponctuent son œuvre témoignent de sa fascination pour le visuel, fascination qui est au cœur même du diptyque que forment les deux premiers ouvrages de sa récente tétralogie. Au-delà de leurs similarités structurelles, ces deux romans illustrent cependant des conceptions contrastées des liens intimes qui unissent langage et vision. Cette étude a pour objet de montrer que de l'exploration de ces contrastes nait, non point une aporie stérile, mais la nécessité d'un « détour » par le pictural. Je m'attacherai donc tout d'abord à examiner les deux parcours que suivent ces romans, afin de mieux pouvoir explorer, dans un second temps, les mystérieuses sentes du «détour» par le pictural que ces contrastes appellent.

Intéressons-nous en premier lieu aux parcours contrastés qu'empruntent ces deux ouvrages. Ils sont encore soulignés par certaines analogies structurelles. Outre leur continuité diégétique, la narration des mésaventures de la famille Potter de 1952 à 1957, les deux romans présentent une structure semblable: ils s'ouvrent sur des prologues proleptiques, respectivement datés de 1968 et 1980, qui ont pour cadre un musée des beaux-arts londonien (The National Portrait Gallery et The Royal Academy of Arts) et leurs interrogations fondamentales se reflètent dans deux pièces de théâtre mises en abyme, qui sont l'œuvre du même dramaturge, Alexander Wedderburn.

3 Sur ces similarités reposent cependant des différences marquées. The Virgin in the Garden a pour cadre l'Angleterre de 1952 au mois de décembre 1953, époque marquée par l'accession au trône d'élisabeth II en 1953, le «Festival of Britain » et l'espoir de voir 
naître une nouvelle période de gloire, un nouvel âge Elisabéthain. Il explore le paradis perdu du règne de la Reine Vierge, et le système de correspondances liant pensée, mots et choses qui le caractérisait, correspondance dont la « dissociation de la sensibilité », telle que la définit T.S. Eliot, nous aurait définitivement privés. Byatt fut fortement influencée par cette théorie, ce qui ne l'empêche d'exprimer une certaine ambivalence à son égard. Cette influence découle notamment de son attrait pour les poètes métaphysiques : « most seductive in Eliot, to me, was his admiration for the metaphysical poets, for their mixture of intellect and passion, sense and sensuousness. My lost paradise was Eliot's elegant fiction of the undissociated sensibility, in which Donne felt his thought as immediately as the odour of a rose ». Son écriture est littéralement hantée par les échos d'un projet de thèse avorté sur l'allégorie religieuse au XVII ${ }^{e}$ siècle, projet d'ailleurs repris par Frederica Potter: « It is not much to say that this unwritten work, with its neoplatonic myths, its interest in the incarnation, in fallen and unfallen (adequate and inadequate) language to describe reality, has haunted both my novels and my reading patterns ever since » (PM 3). The Virgin in the Garden s'inspire de l'analyse de l'épistémè de la Renaissance qu'élabore M. Foucault dans Les Mots et les choses: " Dans son être brut et historique du XVI ${ }^{e}$ siècle, le langage n'est pas un système arbitraire ; il est déposé dans le monde et il en fait partie à la fois parce que les choses elles-mêmes cachent et manifestent leur énigme comme un langage, et parce que les mots se proposent aux hommes comme des choses à déchiffrer ". En Angleterre, la cohésion de ce système de correspondances repose sur la reine Élisabeth I, identifiée par ses contemporains au primum mobile, la sphère extérieure qui, dans le système de Ptolémée, impose son mouvement au reste de l'univers, ainsi que l'explique Tillyard dans The Elizabethan World Picture. The Virgin in the Garden s'intéresse tout particulièrement à la riche iconographie de la Reine Vierge qui s'inspire de la symbolique d'Astrée, personnage tout en nuances: «the phoenix, the rose, the ermine, the Golden Age, the harvest-queen, Virgo-Astrae, virgin patroness of justice and foison ", "she was a whore, of Babylon or London, a clandestine mother, a man, Shakespeare " (VG 13), "Spenser's Dame Nature, who "hath both kinds in one", "nor needeth other none" " (VG 13). "The just virgin is thus a complex character, fertile and barren at the same time; orderly and righteous, yet tinged with oriental moon-ecstasies ", comme le résume Frances Yates. Cet être aux multiples visages occupe une place centrale dans le roman, Astrae est d'ailleurs le titre choisi pour l'œuvre théâtrale mise en abyme.

Par contraste, Still Life reflète le désir d'accéder à « la chose même », dans toute sa nudité, vierge de toute métaphore, de retrouver un langage qui serait en mesure de simplement dénoter, d'être purement mimétique. Cette quête s'apparente à la volonté de nommer et de classifier qui caractérisait la pensée classique, ainsi qu'à la recherche flaubertienne du «mot juste ». Le symbole en est la chaise jaune de Van Gogh, qui semble pouvoir désigner la chose même :

I wanted to write about birth, about death, plainly and exactly. There were ideological reasons for this, as well as a sense that my novel must move from an undissociated paradise to our modern dissociated world. I wanted both to demythologise my novel and to describe the demythologising of the Church in the novel. I am afraid of, and fascinated by, theories of language as a self-referring system of signs, which doesn't touch the world. I am afraid of, and resistant to, artistic stances which say we explore only our own subjectivity. [...] I am also resistant to the idea that the world hits us as a series of random impressions (V. Woolf) and that memory operates in a random manner. I wanted at least to work on the assumption that order is more interesting than the idea of the random (even if 
our capacity to apprehend it is limited): that accuracy of description is possible and valuable. That words denote things. (PM 11)

5 Les deux pièces de théâtre mises en abyme s'inspirent de tableaux: le célèbre portrait d'élisabeth I connu sous le nom de "The Darnley Portrait » et The Yellow Chair (ou La Chaise de Vincent) de Van Gogh. Ces deux emblèmes opposés, la Reine Vierge et l'humble chaise jaune de Vincent, reflètent les contrastes des deux œuvres.

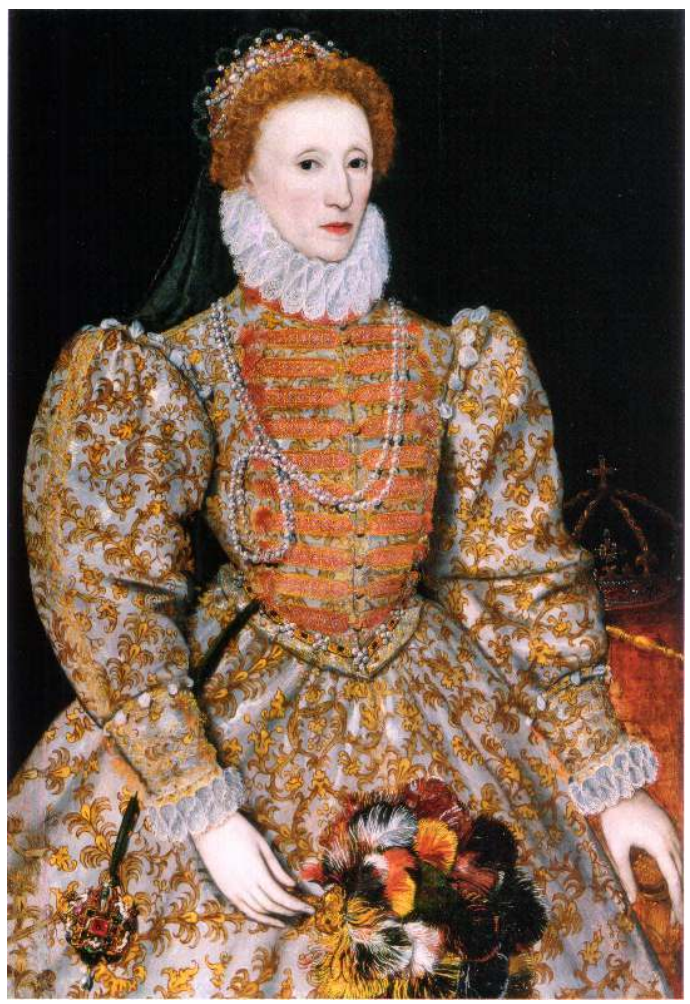

The «Darnley Portrait » of Elizabeth I of England (c. 1575), huile sur bois, $113 \times 78,7 \mathrm{~cm}$, National Portrait Gallery, Londres, Creative Commons 


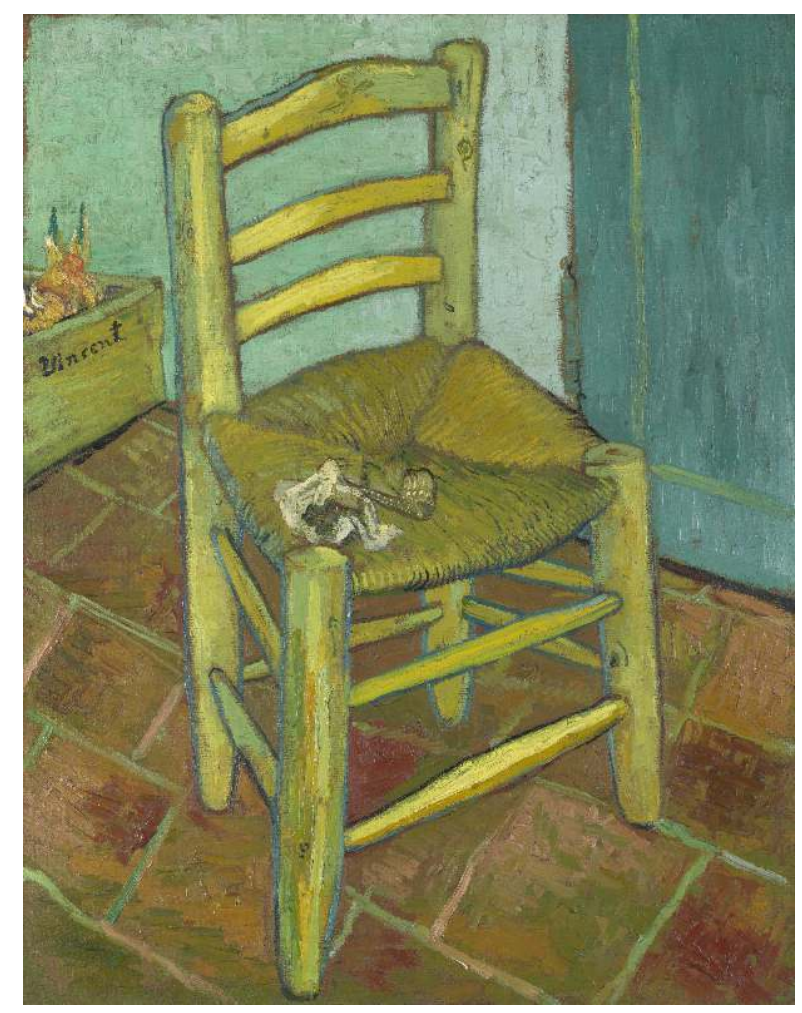

Vincent Van Gogh, La Chaise de Vincent (1888), huile sur toile, 91,8x73cm, National Gallery, Londres, Creative Commons

Ces oppositions se retrouvent également dans les genres picturaux évoqués par leurs titres. The Virgin in the Garden introduit l'ébauche d'une structure narrative fondée sur les deux articles définis, qui offrent un ancrage spatial et subjectif propice au développement d'un récit, et sur un horizon d'attente de type binaire, qui appelle son complémentaire, la tentation. Ce titre évoque une peinture allégorique. Still Life revendique au contraire une certaine parenté avec un genre pictural autre, associé dans les appellations française et anglo-saxonne à la mort ou à la vie silencieuse et qui semble par conséquent aller à l'encontre de toute élaboration romanesque, de toute intrigue.

7 Les deux œuvres expriment un même désir de retrouver une certaine coïncidence, une adéquation, un contact intime des mots et des choses, auquel il semble cependant impossible d'accéder dans notre monde moderne dissocié. Ces deux démarches herméneutiques, associées à des réflexions sur le pictural et sur les épistémès d'autres âges, offrent un commentaire sur la crise de la représentation moderne. La béance générée par la crise du sens donne lieu soit à la poursuite du "démon de l'analogie », à la prolifération anarchique des liens relationnels, soit à la quête de la dénotation pure, de la transitivité, de l'immanence. Cette oscillation du langage entre pléthore et manque, transparence et obstacle, affirmation ou déni de son pouvoir mimétique, n'entraîne cependant pas une aporie, mais un paradoxal « détour » par le pictural, par la médiation de l'hétérogène, pour essayer de retrouver proximité et immédiateté, détour qui mêle considérations esthétiques et épistémologiques. L'écriture de Still Life ne s'est, bien entendu, pas conformée au projet initial de suppression totale de la métaphore. La chaise de Vincent elle-même se révèle être une métaphore complexe, qui mêle des considérations psychologiques, culturelles, religieuses et esthétiques, et Byatt souligne l'impossibilité de penser, voir, écrire et même nommer sans métaphore. Il s'agissait 
plutôt d'explorer toutes les possibilités mimétiques du langage, et des contrastes que cette exploration a révélés est née la nécessité d'un détour par le pictural, toujours présent dans l'œuvre de Byatt, mais qui devient véritablement le noyau de Still Life, roman plus expérimental qui multiplie les considérations théoriques et métafictionnelles.

Nous arrivons donc au second temps de cette étude, l'exploration du « détour " par le pictural. Cet appel à un système de signes hétérogène se fait dans un esprit de fertilisation réciproque, d'échange, mais aussi de respect de la différence. Les incipits, le dialogue instauré entre les références picturales et l'écriture romanesque, et les points de croisement que sont les métaphores et les visions sont ici au cœur de l'inscription du pictural.

9 Les deux incipits se déroulent dans un musée des beaux-arts, ce qui a pour effet de placer les deux ouvrages à proprement parler dans le cadre du pictural, qui se voit ainsi attribué une valeur non seulement thématique, mais structurelle et symbolique, à l'intérieur d'un texte-seuil qui est tout à la fois dans l'œuvre et hors de l'œuvre, produisant ce que Derrida nomme l'effet-parergon. Le musée est le lieu de la célébration du pictural par excellence, mais aussi, plus généralement, le lieu de la mise en scène, de la monstration du regard. Il se prête tout particulièrement à l'ekphrasis, et permet d'introduire judicieusement le thème de la mise en regard de l'image et du texte, par de multiples biais: pièce de théâtre, catalogues d'exposition, ekphrasis et commentaires de personnages centraux, les gens du livre que sont Frederica Potter et Alexander Wedderburn. D'autre part, le musée est un lieu public dont la fonction est de célébrer le sens esthétique, le regard, de l'observation critique à l'admiration muette, et un lieu de prestige où l'on vient souvent tant pour voir que pour se faire voir. Frederica et Alexander aiment à s'y donner en spectacle: "They were beginning to attract a penumbra of gallery-goers, as though they were offering a guided tour ». La volonté de faire du regard la source et le fondement des deux romans est encore soulignée par l'apparition furtive de Byatt elle-même dans chacun des prologues. Au début de The Virgin in the Garden, lors du spectacle dans lequel Flora Robson interprète Élisabeth I à la National Gallery en 1968, se trouve dans l'audience Antonia Fraser, accompagnée d'une femme, « a dumpy woman in a raincoat " (VG 11) que l'on peut identifier à l'auteur. Une figure similaire apparait dans le prologue de Still Life, lors de l'exposition postimpressionniste de 1980 à la Royal Academy. L'organisateur de l'exposition présente à Frederica « a smallish woman in a pine-green tent-like coat » (SL 3) dont il bredouille le nom et dont il est dit que Frederica la considère à tort comme historienne de l'art. L'inconnue observe la jeune Potter, "with an apparently absent-minded scanning attention" (SL 4). À l'appui de ces identifications, M. Westlake cite Byatt, qui aurait affirmé lors d'une conférence sur l'identité en 1986: «My self is not contained in the story, but it is contained in the person scanning ». La deuxième apparition confirme, si besoin était, que Byatt entend placer les diverses modalités du voir au centre de sa création artistique.

Ces prologues contiennent en germe les principaux thèmes des romans, qui seront particulièrement développés dans Still Life. Si le premier roman de la tétralogie témoigne de l'écriture sensuelle de Byatt et contient des descriptions fines et remarquables telles les visions de Marcus Potter ou la promenade de Daniel Orton et Stephanie Potter sur la plage de Filey, dans Still Life, les descriptions sont très fréquemment accompagnées d'une intervention du narrateur introduisant des réflexions sur la notion de représentation, nourries par de multiples références à des œuvres picturales et à des artistes aussi divers 
que Vermeer, Monet, Xavier Mellery, Turner, Gauguin, Cézanne, Matisse, Picasso, Mondrian, Jackson Pollock et, bien entendu, Van Gogh. Ces «digressions » sont indissociables de la diégèse, qui les engendre et qu'elles influencent en retour, instaurant un constant dialogue, au sein duquel nous privilégierons ici trois thèmes essentiels, l'expression de l'étranger, la quête d'immédiateté et la représentation des couleurs, thèmes particulièrement développés au sein des chapitres 4 (61-74), 7 (103-132) et 14 (193-211), respectivement intitulés « Midi », « A Birth » et « Figures of Speech ». Lorsque Frederica, enchantée par la découverte des paysages du Sud de la France, exprime le désir de devenir écrivain, le narrateur médite sur l'évocation artistique de l'étranger, sur les nombreux voyages des peintres (Monet au Cap d'Antibes, Turner à Venise ou Gauguin à Tahiti) et évoque les particularités des différents média artistiques : «Pigment is pigment and light is light in any culture. But words, acquired slowly over a lifetime, are part of a different set of perceptions of the world, they have grown with us, they restrict what we see and how we see it " (SL 72). Si les pigments, comme la lumière, ont une existence propre, indépendante de l'homme, le langage est un produit culturel, il fait corps avec la pensée et tend à orienter la perception, ramenant l'inconnu au connu. Cette réflexion se poursuit dans le cadre du second passage mentionné. Le prisme du langage nous éloigne de la singularité du réel, il faut se méfier de sa transparence illusoire :

Paint itself declares itself as a force of analogy and connection, a kind of metaphormaking between the flat surface of purple pigment and yellow pigment and the statement "This is a plum." "This is a lemon." "This is a chair." "This is a breakfast table." [...] It is impossible not to think about the distance between paint and things, between paint and life, between paint and the 'real world' (which includes other paintings). [...] It is not at all impossible, it is even common, not to think about the distance between words and things, between words and life, between words and reality. (SL 200-201)

11 La peinture, par la présence d'une toile, d'un cadre, de pigments, s'affirme d'emblée comme représentation, tandis que l'espace du langage est celui de la communication interindividuelle "words are our common currency » (SL 201). Cette mise en regard du littéraire et du pictural, qui implique une redécouverte de la matière même du langage, est le plus souvent accompagnée d'une référence à Van Gogh, qui occupe une place essentielle dans Still Life, et ce, à de multiples points de vue. À l'origine et au centre du dialogue texte-image se trouve la correspondance du peintre, d'un homme lettré à la sensibilité exacerbée qui mène notamment des réflexions profondes et passionnantes sur la création artistique et la représentation, et nous offre de précieux exemples d'ekphrasis. Son œuvre picturale est la source de nombreuses considérations sur la représentation littéraire et sur la quête d'immédiateté qui fonde Still Life: "Provence is as he painted it, we use his images as icons by which to recognise certain things, the cypresses above all, the olive, some configuration of rock and vegetation, the line of the Alpilles, the plain of Crau, the light itself » (SL 74). Les images de Van Gogh en viennent à représenter si parfaitement et si singulièrement leur objet qu'il semble qu'elles le font véritablement voir et reconnaître, telles des icônes, signes dont le signifié et le signifiant sont dans une relation "naturelle» de ressemblance, selon la terminologie de Peirce. Le choix d'un modèle tel que Van Gogh, dont la touche et les couleurs, si singulières et expressives, ne sauraient être assimilées à un réalisme mimétique, peut sembler paradoxal dans le cadre d'une volonté d'atteindre "la chose même ». La représentation ne saurait cependant copier le réel. Elle le donne à voir autrement, à travers le prisme d'une conscience individuelle. Paradoxalement, la touche si originale de Van Gogh semble au plus près du sensible. A.S. Byatt s'applique tout particulièrement à décrire cette touche singulière : 
The brushstrokes in the 'Sower' are almost tesselations, the sky flows with them, the furrows of purple earth run away from the central heavy gold sun, the sower scatters seeds of gold light which are brushstrokes in the pattern on the dark morning clods. They are thick and solid: they are the movement of light over things, of the eye over things. In "The Reaper" Vincent's later spiralling forms are everywhere, curling and linking the white-hot furnace of the cornfield, the blue figure of the man, the purple mountains, the green air, into one substance, his vision. (SL 132)

La matière même de la peinture impose sa mystérieuse présence et rend manifeste l'acte de perception, les mouvements de l'œil et l'unité profonde de la représentation autour d'une vision. À la suite de cette description, les réflexions métafictionnelles se multiplieront, quittant le domaine du pictural pour s'attacher au grain du langage, au mystère de sa matérialité, à la richesse de sa texture, notamment dans le chapitre 27 « Names of Grasses ». Le dire devient objet du regard. Byatt, à l'instar de Iris Murdoch, aime à considérer les mots comme un filet dont l'homme couvre les choses afin d'en épouser les contours. L'affinement de la texture de ce filet, l'attention apportée à son tissage, par l'intermédiaire d'une réflexion sur la représentation et le pictural sont un prélude à la redécouverte du réel. Mais si toute représentation est interprétation, toute perception est également le fruit d'une activité interprétative, comme le montre l'interrogation sur une possible innocence du regard générée par la naissance du premier enfant de Stephanie Potter :

Art is not the recovery of the innocent eye, which is inaccessible. "Make it new" cannot mean, see it free of all learned frames and names, for paradoxically, it is only a precise use of learned comparisons and the signs we have made to distinguish things seen or recognised that can give the illusion of newness. [...] [O]ne cannot think at all without a recognition and realignment of ways of thinking and seeing we have learned over time. We all remake the world as we see it, as we look at it. (SL 131)

13 Toute perception est interprétation, toute représentation est interprétation de cette perception, et s'inscrit dans diverses traditions, tant en littérature qu'en peinture. Les représentations de Van Gogh illustrent le rapport entre la tradition et le talent individuel et font ainsi écho aux fréquents dialogues intertextuels qui sont au cœur des œuvres de Byatt :

He expected to see "Japanese" subjects, the colours of Monticelli, the forms of Cézanne and Renoir, the southern light lauded by Gauguin as a mystic necessity. He saw all these things, as he expected them. He saw also Dutch things in the French heat, bridges not formally different from those in Delft and Leyden, colours in the glare that reminded him primarily of the soft blues and yellows of Vermeer. Also, and simultaneously, he saw what no one had yet seen, what was his to see. Sunflowers, cypresses, olives. (SL 74)

La peinture de Van Gogh est célébrée pour sa touche si particulière, mais aussi pour l'expressivité de ses couleurs. Dans sa correspondance, Van Gogh n'accorde que rarement les adjectifs de couleurs avec les noms qu'ils qualifient. Les couleurs semblent ainsi acquérir une autonomie, et, paradoxalement, plus de réalité que les choses: «The result was that they could almost be read as things more real than the things they qualified, a pattern of eternal forms from another world, not part of the solid world of cabbages and pears - yellow and violet, blue and orange, red and green » (SL 97). Van Gogh est fasciné par la "métaphysique des couleurs ", leur mariage mystique, le "noir-rouge ", l'éclat de la lumière et l'intensité de l'azur sous le soleil méditerranéen. Et il fait souvent part de cette fascination dans ses lettres, fascination que partage Byatt, qui s'interroge sur 
l'apparente impuissance des mots à dire ces sensations élémentaires. Lors d'un petitdéjeuner chez la famille Poole, le dramaturge Alexander Wedderburn s'émerveille de la nature morte, singulièrement animée, que forment le beurre, le müesli, les fruits et le yogourt. "The breakfast table was a still life, with the easy life of vegetables and culture » (SL 198). Alexander songe au tableau de Van Gogh qui figure en couverture de l'édition Vintage et que Byatt nomme The Breakfast Table, un tableau qui l'obsède « a clean, bright paradox, still and very much alive, held together by the contrast and coherence of blue and yellow »(SL 202). Lorsque Vincent le décrit à son frère Théo, il souligne qu'il contient six bleus différents, et quatre ou cinq sortes de jaune et d'orange : «In Alexander's mind these colour-words sang like a poem » (SL 202). Le roman Still Life témoigne d'un attrait profond pour la poésie de la nature morte, telle que Charles Sterling la célèbre dans $L a$ Nature Morte de l'Antiquité à nos jours :

Si on ne se lasse pas d'une nature morte comme on se lasse d'un portrait ou d'une composition, ce n'est pas seulement parce que le jeu des lignes et des couleurs y est entièrement libéré de l'anecdote et des précises suggestions du sujet que nous finissons par épuiser. Ce n'est pas seulement par la latitude que la nature morte laisse à notre imagination, mais bien parce que les choses inanimées, si étroitement mêlées à nos jours, représentent le commerce le plus immédiat de l'homme avec la matière. C'est par elles que chacun de nous - enfant au berceau, palpant les objets pendant des heures - a pris les premiers contacts avec le monde, et l'artiste nous rend en elles nos premiers étonnements et nos premiers rêves.

Suite à l'observation de cette nature morte, le narrateur se fait le relais des questionnements qu'engendre le désir qu'éprouve Alexander de décrire précisément la couleur d'une prune. Cette interrogation, qui peut sembler triviale, dissimule des profondeurs insoupçonnées et touche au cœur même de la relation du langage au réel. Byatt, après Flaubert, recherche "le mot juste ", «the exact word » capable de rendre compte de cette couleur particulière. Cette quête du "mot juste» se situe dans le prolongement de la représentation comme nécessité humaine. Cette nécessité naît du besoin d'exprimer la reconnaissance d'un équilibre, d'une plaisante harmonie qui répondent à une quête essentielle de l'homme. La représentation permet de conserver et de comprendre l'éphémère aperçu de cette harmonie. Elle implique un investissement subjectif considérable, une attention curieuse, qui seuls permettent de véritablement comprendre l'objet. La quête de l'expression adéquate se heurte toutefois à l'impression que la perception visuelle est infiniment plus riche, précise et nuancée que ne peut le décrire un terme de couleur. Une observation attentive met régulièrement à l'épreuve les codes langagiers. L'expression, condamnée à utiliser un petit nombre de termes inadéquats, réduit ou fausse l'expérience première. Un des obstacles majeurs réside dans l'insuffisance des noms ou des adjectifs: «there are shepherds in the Andes who have over sixty words for the colour brown in the coats of sheep. But they are shepherds in the Andes » (SL 72). L'expression du violet de la prune et de ses nuances ne peut exploiter dans la langue anglaise une telle richesse nominale: "What is the greyish, or maybe white, or whitish, or silvery, or dusty mist or haze or smokiness over the purple shine? How do you describe the dark cleft from stalk-pit to oval end, its inky shadow? (SL 199). La description exacte, le rendu fidèle d'un objet précis sont impossibles, ne serait-ce que parce que le nom générique n'évoque pas une abstraction, mais une image particulière, éminemment variable d'un lecteur à l'autre. Afin de partager l'image d'un fruit particulier, l'écrivain doit par conséquent employer des adjectifs précis et évocateurs :

A writer aiming for unadorned immediacy might say: a plum, a pear, an apple, and

by naming these things evoke in every reader's mind a different plum, a dull 
tomato-and-green specked Victoria, a yellow-buff globular plum, a tight, blackgreen damson. If he wishes to share a vision of a specific plum he must exclude and evoke: a matt, oval, purple-black plum, with a pronounced cleft. (SL 199) descriptive des termes, l'évocation du velouté mat de la prune " glistening, overlaid with a matt softness " par le terme "bloom », la description de la texture ferme de la chair n'excluent pas leur très forte valeur métaphorique. La richesse suggestive des signifiants se déploie dans l'esprit de l'écrivain comme du lecteur: «But you cannot exclude from the busy automatically-connecting mind possible metaphors, human flesh for fruit flesh, flower-bloom, skin bloom, bloom of ripe youth for this powdery haze, human clefts, declivities, cleavages for that plain noun » (SL 199). Paradoxalement, cette prolifération des métaphores permet toutefois de se rapprocher de la chose, ou plutôt d'être au plus près de l'acte de percevoir, de l'appréhension humaine du réel. L'évocation suggestive donne à la prune une vie, une présence insoupçonnées. L'ineffable couleur de la prune est finalement assimilée, avec une remarquable précision et dans une métaphore qui associe plaisir et meurtrissure, au sombre centre d'une ecchymose naissante « the dark centre of some new and vigorously burgeoning human bruise » (SL 199). La fonction référentielle du langage est plus métaphorique que mimétique: «Language might relate the plum to the night sky, or to certain ways of seeing a burning coal, or to a soft case enwrapping a hard nugget of treasure. Or it might introduce an abstraction, a reflection, of mind, not mirror » (SL 200). La réflexion est plus spéculative que spéculaire, elle reflète l'esprit plus que le monde, ou plutôt le monde vu au filtre de l'esprit, et non mimétiquement reproduit. La métaphore intensifie notre présence au monde, notre investissement subjectif.

Ces réflexions nous conduisent au dernier point de cette seconde partie, à savoir une évocation des métaphores et visions, qui illustrent le rôle essentiel de l'image dans l'écriture de Byatt. La métaphore est à la source même de la structure de ses œuvres et de leur thématique: «In my experience I know what the form of a novel is when I find what I think of as the "ruling metaphor" » (PM 9-10). Elle conçoit la genèse de ses œuvres en termes de lignes, de couleurs et de formes et visualise toute métaphore :

I don't know how much is known about the difference between those who think with mental imagery and those who don't. I very much do - I see any projected piece of writing or work as a geometric structure: various colours and patterns. I see other people's metaphors - if there is an iconic content to a metaphor I will "see" a visual image on some inner mental screen, which can then be contemplated more precisely, described discursively. (PM 13-14)

Les ensembles chromatiques originels étaient rouges, blancs et verts pour The Virgin in the Garden et jaunes et violets pour Still Life. La métaphore centrale de The Virgin in the Garden était une métaphore de métamorphose, de chair en pierre ou en herbe, des passions humaines en art (l'urne grecque de Keats et la statue d'Hermione dans A Winter's Tale), du langage en fleur. Cette dernière métamorphose est indissociable d'une œuvre picturale, le Printemps de Botticelli. Le langage «fleuri » est associé au flot de fleurs qui s'écoule de la bouche de la nymphe Chloris. Ces fleurs sont par la suite brodées sur sa robe, lorsqu'elle est devenue Flore, l'épouse de Zéphyr. Elles sont issues de la terre et la fécondent en retour. Le langage habille la pensée et semble tout à la fois participer de la substance des choses. Ces multiples fleurs, et notamment les roses rouges et blanches que Flore transporte dans un repli de sa robe, sont également associées aux roses de York et 
Lancaster. Par contraste, la graine poétique à l'origine du jardin littéraire de Still Life est évoquée en ces termes :

The germ of this novel was a fact which was also a metaphor: a young woman, with a child, looking at a tray of earth in which unthinned seedlings on etiolated pale stalks died in the struggle for survival. She held in her hand the picture of a flower, the seed packet with its bright image. Nasturtium, Giant Climbing, mixed. (SL 286-287)

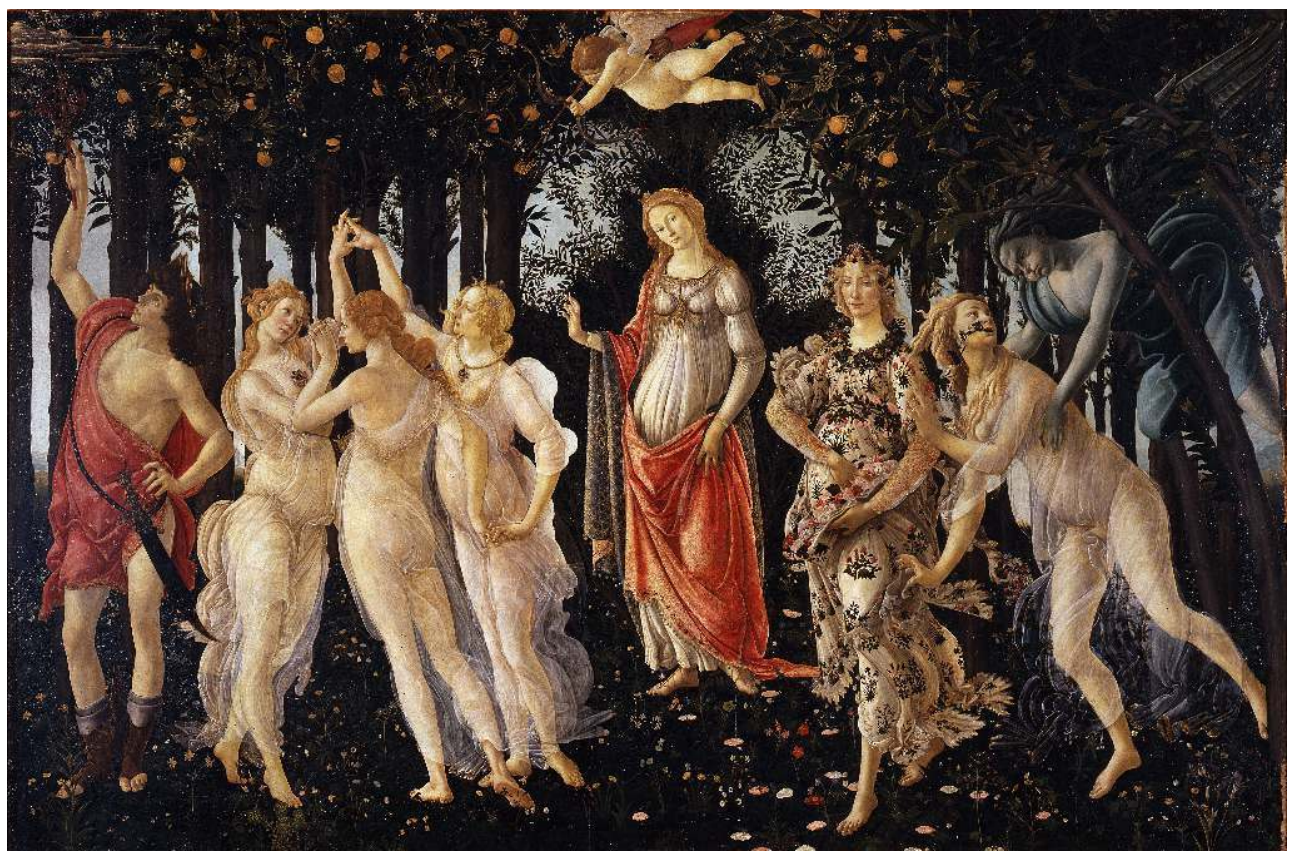

Sandro Botticelli, La Primavera (1482), tempera, 203×314cm, Galerie des Offices, Florence, Creative Commons

19 La métaphore reste florale, mais à la fertilité prolifique, à l'éclosion multiple et à la floraison se substituent la simplicité et la fragilité de la germination. Il s'agit de revenir aux sources mêmes de la nomination et des figures de style. Au tableau de Botticelli succède l'image d'un paquet de graines illustré représentant le désir de dénoter le réel, d'être au plus près de la chose même. Still Life, roman dont le projet était de renoncer à toute métaphore, est devenu un ouvrage sur la métaphore, sur le " voir comme », selon la belle expression de Ricœur dans La Métaphore vive. Située entre pensée et expérience, entre sens et image, la métaphore est à la fois une expérience et un acte. Elle révèle « la dynamique à l'œuvre dans la constitution des champs sémantiques [...] [en] présent[ant] de manière ouverte, par le moyen d'un conflit entre identité et différence, le procès qui, de manière couverte, engendre les aires sémantiques par fusion des différences dans l'identité ». Still Life reproduit les tensions inhérentes à cette figure dans de multiples contrastes internes et cherche à dévoiler les processus à l'origine de la représentation. Son projet initial de décrire «birth and death, plainly and exactly » (PM 11), de transcrire la vie singulière des natures mortes et d'atteindre «la chose même » conduit l'écriture à emprunter de multiples détours, à adopter l'oblique et l'indirection afin de pouvoir évoquer l'ineffable.

Cette volonté de dire l'ineffable naît également d'une certaine affinité avec des personnages "visionnaires» tels que Van Gogh, Samuel Palmer, Coleridge, Blake ou, parmi les créations fictionnelles de Byatt, Henri Severell et Marcus Potter. Chacun des deux romans s'attache à l'une de ces figures. Dans The Virgin in the Garden, Marcus Potter, 
jeune garçon asthmatique et petit génie mathématique, est assailli par d'intenses visions lumineuses qui le mènent au bord de la folie. Dépourvu de sens religieux et de talents artistiques, il n'a pour seule défense que la géométrie, et les interprétations délirantes de son professeur de biologie, qui le croit capable d'entrer en communication avec la noossphère. Still Life explore comment Van Gogh combattit sa folie en tentant de donner forme et sens à ses visions par la peinture: «He ordered his world of raw and sophisticated vision with the mapping, the patterning of his brushstrokes, with the more primitive sense of touch » (SL 132). Parallèlement, le roman s'interroge sur la possibilité et la manière d'écrire ces visions, notamment à travers l'exemple de la pièce mise en abyme. C'est dans le cadre de cette réflexion sur la représentation, indissociable d'une redécouverte de la matière, de la "chose même ", que Marcus parvient finalement à surmonter ses peurs et à se délivrer de ses visions d'horreur, notamment en se découvrant une passion pour la biologie, qui culmine dans un véritable sentiment d'osmose avec la nature (ch. 28, «A Tree, of Many, One »). Byatt place ces phénomènes de visions à la source même de sa créativité : « Henri Severell is partly simply my secret self [...]. Someone who saw everything too bright, too fierce, too much [...]. This vision of too much makes the visionary wants to write - in my case - or paint, or compose, or dance, or sing ».

21 C'est en des couleurs vives et lumineuses que Byatt dépeint l'intimité de son paysage intérieur d'écrivain, comme le montrent ses commentaires à l'égard de son portrait officiel. Celui-ci fut exécuté à sa demande par un peintre abstrait et un grand coloriste, Patrick Heron. Exposé à la National Portrait Gallery de Londres, il porte l'intitulé suivant : « Dame Antonia Susan ('A.S.') Byatt. Red, Yellow, Green and Blue : 24 September 1997 ». Le visage de l'écrivain est figuré par quelques traits simples mais évocateurs, qui se détachent sur un fond composé de grands à-plats de couleurs primaires. La simplicité des contours et la vivacité des couleurs peuvent sembler en contradiction avec l'art d'un écrivain célébré pour la minutie de son réalisme et son évocation de l'«anglicité ». A.S. Byatt reconnaît toutefois en ce portrait l'expression profonde de sa vision d'écrivain :

The energy, the brashness, the uncompromising splashes of primary colour represented what I wanted in an abstract portrait by a great colourist. But they represented something else as well. They were a painting of the writer, of how I feel when I start work, a vanishing, watching body in a sea of light and brilliance.

Le début du processus créatif se manifeste pour A.S. Byatt par un évanouissement du corps ("vanishing»), qui semble devenir impersonnel et se concentre en un regard curieux (" watching »), qui baigne dans un océan de lumière, un chaos informe de lumière liquide observé du coin de l'œil et qui progressivement prend forme. Ce portrait abstrait est un portrait extrêmement intime de l'écrivain, une vision interne : «He had somehow drawn the body as it feels from inside, looking out. The watcher watched ».

Si Byatt ne dissimule pas son admiration pour le pictural, qui se manifeste notamment par les nombreux articles qu'elle a publiés dans des revues artistiques, elle privilégie toutefois l'imagination créatrice, l'investissement imaginatif qu'implique l'image verbale, la description littéraire, "the energy which is generated by the visualised unseen », ainsi qu'elle l'explique dans Portraits in Fiction. Passionnée par les liens conflictuels qui unissent les mots et les choses, Byatt célèbre le pouvoir créateur de la «métaphore vive ». Le "détour " par le pictural que les contrastes de ces deux romans appellent nourrit la réflexion métafictionnelle, interroge le statut et les formes de la représentation. Ce détour est pour Byatt véritablement essentiel et permet un retour enrichi à la littérature. 
Cette ouverture à l'hétérogène entraîne une redécouverte du grain du langage, de sa texture propre, de ses riches potentialités, au-delà de la stérile opposition de la transparence et de l'obstacle, et favorise une plus grande perméabilité au monde.

INDEX

oeuvrecitee Virgin in the Garden (The), Still Life 